\title{
Identifying the Environment and Redshift of Gamma\#Ray Burst Afterglows from the Time Dependence of Their Absorption Spectra
}

\section{Citation}

Perna, Rosalba, and Abraham Loeb. 1998. "Identifying the Environment and Redshift of Gamma\#Ray Burst Afterglows from the Time Dependence of Their Absorption Spectra." The Astrophysical Journal 501 (2): 467-72. https://doi.org/10.1086/305865.

\section{Permanent link}

http://nrs.harvard.edu/urn-3:HUL.InstRepos:41393218

\section{Terms of Use}

This article was downloaded from Harvard University's DASH repository, and is made available under the terms and conditions applicable to Other Posted Material, as set forth at http:// nrs.harvard.edu/urn-3:HUL.InstRepos:dash.current.terms-of-use\#LAA

\section{Share Your Story}

The Harvard community has made this article openly available. Please share how this access benefits you. Submit a story. 
THE Astrophysical JournaL, 501:467-472, 1998 July 10

(C) 1998. The American Astronomical Society. All rights reserved. Printed in U.S.A.

\title{
IDENTIFYING THE ENVIRONMENT AND REDSHIFT OF GAMMA-RAY BURST AFTERGLOWS FROM THE TIME DEPENDENCE OF THEIR ABSORPTION SPECTRA
}

\author{
Rosalba Perna and Abraham Loeb \\ Harvard-Smithsonian Center for Astrophysics, 60 Garden Street, Cambridge, MA 02138 \\ Received 1997 December 3; accepted 1998 February 16
}

\begin{abstract}
The discovery of gamma-ray burst (GRB) afterglows revealed a new class of variable sources at optical and radio wavelengths. At present, the environment and precise redshift of the detected afterglows are still unknown. We show that if a GRB source resides in a compact ( $\lesssim 100 \mathrm{pc}$ ) gas-rich environment, the afterglow spectrum will show time-dependent absorption features due to the gradual ionization of the surrounding medium by the afterglow radiation. Detection of this time dependence can be used to constrain the size and density of the surrounding gaseous system. For example, the $\mathrm{Mg}$ II absorption line detected in GRB 970508 should have weakened considerably during the first month if the absorption occurred in a gas cloud of size $\lesssim 100 \mathrm{pc}$ around the source. The time-dependent $\mathrm{H}$ I or metal absorption features provide a precise determination of the GRB redshift.
\end{abstract}

Subject headings: cosmology: theory — gamma rays: bursts

\section{INTRODUCTION}

The detection of delayed emission in X-ray (Costa et al. 1997), optical (van Paradijs et al. 1997; Bond 1997), and radio (Frail et al. 1997) wavelengths following gamma-ray bursts (GRBs), the so-called afterglows, can be reasonably explained by models in which the bursts are produced by relativistically expanding fireballs (Paczyński \& Rhoads 1993; Meszaros \& Rees 1997; Vietri 1997a; Waxman 1997a, 1997b; Wijers, Rees, \& Meszaros 1997b; Vietri 1997b; Sari 1997). On encountering an external medium, the relativistic shell that emitted the initial GRB decelerates and converts its bulk kinetic energy to synchrotron radiation, giving rise to the afterglow. Since the emission occurs on time and length scales that are much larger than those of the initial explosion, the dynamics of the expanding shell is self-similar and thus insensitive to the properties of the energy source (Blandford \& McKee 1976). The source size inferred from radio scintillation data of GRB 970508 (Frail et al. 1997; Taylor et al. 1997) is consistent with the generic prediction of this model (Goodman 1997; Waxman, Kulkarni, \& Frail 1998). Moreover, synchrotron emission by Fermiaccelerated electrons behind the expanding shock can successfully explain the gross properties of the time-dependent spectra observed in the afterglows of GRB 970228 and GRB 970508 for a fireball energy of $\sim 10^{52}$ ergs (Waxman 1997a, 1997b; Wijers et al. 1997a; Sari, Piran, \& Narayan 1997).

In the simplest emission model, the afterglow flux is proportional to the square root of the ambient medium density (e.g., Waxman 1997a), and could therefore span a dynamic range of more than five decades if the ambient density varies between its characteristic values in dense molecular clouds $\left(\sim 10^{4} \mathrm{~cm}^{-3}\right)$ and in the intergalactic medium $\left(\sim 10^{-6}\right.$ $\mathrm{cm}^{-3}$ ). Even merely galactic environments span a wide range of possible gas densities, between those of ellipticals and spirals. The possibility that GRB sources reside in both gas-rich and gas-poor environments might account for the absence of afterglow emission in some GRBs (e.g., Groot et al. 1998). Any direct observational verification of the postulated connection between the afterglow flux and the GRB environment would provide a crucial test of the basic fireball model.
Despite the expectation that bright afterglow sources should reside in gas-rich environments, there is currently no direct measurement of the density, size, or redshift of the host system for known afterglows. The discovery of absorption lines in the optical afterglow of GRB 970508 provided the first direct estimate of source distance, constraining its redshift to the range $0.835 \leq z \lesssim 2.3$ (Metzger et al. 1997; Djorgovski et al. 1997). The possible existence of host galaxies has yet to be conclusively determined from direct imaging of afterglow fields (Sahu et al. 1997; Fruchter et al. 1997; Pian et al. 1997).

If a GRB source resides within the $\mathrm{H}$ I disk of a spiral galaxy, its optical-UV spectrum is likely to show a Lymanlimit trough, a damped Ly $\alpha$ absorption, and metal absorption lines, all at the source redshift. However, the same absorption features could also be produced by an intervening galaxy along the line of sight to the source. The ambiguity between the GRB host and an interloper could be removed by detecting the Ly $\alpha$ forest, which should end at the true source redshift. However, for source redshift $z \lesssim 2$, such a spectroscopic detection cannot be made with ground-based telescopes, requiring the use of the Hubble Space Telescope (hence being feasible only for bright afterglows, because of the small collecting area). In this paper we argue that if the absorption occurs in a sufficiently compact region $(\lesssim 100 \mathrm{pc})$ around the GRB source itself, then the afterglow radiation will ionize the region and change the observed absorption features with time. The release of $\sim 3 \times 10^{51}$ ergs in ionizing photons could ionize $\sim 10^{62}$ hydrogen atoms (or $\sim 10^{5} M_{\odot}$ ) and create an ionized bubble of radius $\sim 100 \mathrm{pc} n_{1}^{-1 / 3}$ in the surrounding galaxy, where $n_{1}$ is the ambient proton density in $\mathrm{cm}^{-3}$. Given the typical $\mathrm{H}$ I column densities found in galactic disks (Broeils \& van Woerden 1994), such a bubble could be big enough to poke a hole in the disk and eliminate the absorption features of the host altogether. Aside from establishing the GRB redshift, detection of this time-dependent effect could be used to constrain the gas density and the size of the GRB host. GRB afterglows are much more effective at ionizing their environments than supernovae, because of their considerably higher UV luminosity. 

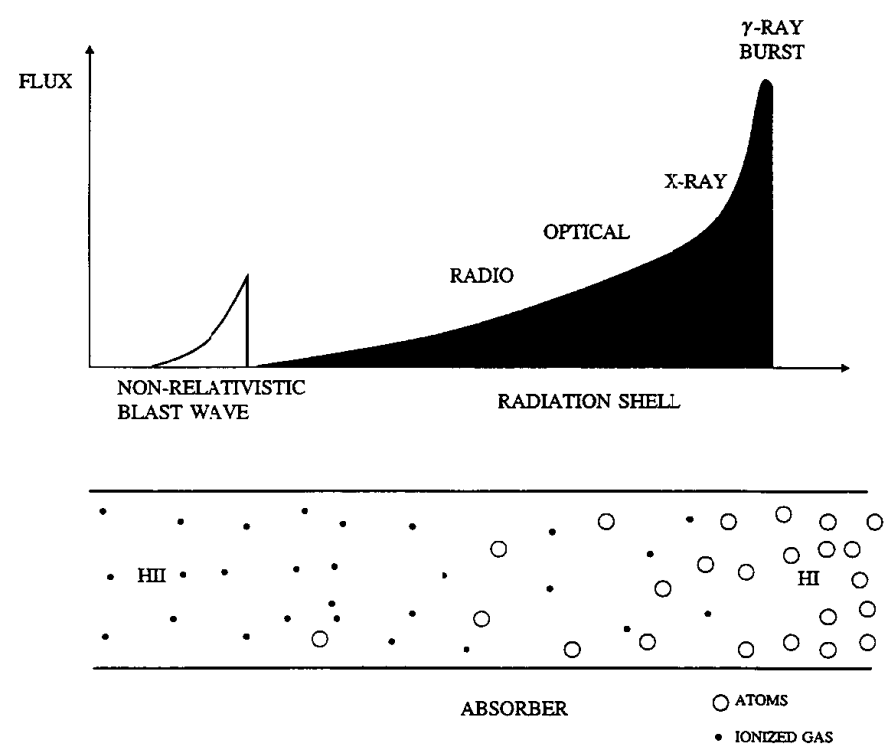

FIG. 1.-Snapshot of the outward-propagating radiation shell and its effect on the ambient absorbing medium. The ambient gas is gradually ionized by the passing UV radiation. The column of absorbing atoms seen by the front of the radiation shell is higher than that seen by the later radiation. As a result, the equivalent width of the absorption lines declines with time from the point of view of a distant observer.

The computation of the time dependence of the absorption spectrum is a nontrivial problem. ${ }^{1}$ A snapshot of the ionizing effect of the radiation shell on the ambient medium is schematically illustrated in Figure 1. At the late times under consideration, the blast wave that produced this radiation is already nonrelativistic and lags behind the radiation front. Only the front of the afterglow light pulse moves into the unperturbed gas. Each of the subsequent radiation shells propagates into a medium that had already been partially ionized by prior shells. Since each radiation shell has a different spectrum at emission and a flux that declines with radius as $1 / r^{2}$, the ionization state of the ambient medium is generally inhomogeneous and time dependent. The eventual absorption spectrum seen by a distant observer can be calculated by coupling the radiative transfer equation to the radiative ionization equation for the medium. In $\S 2$, we formulate the set of causal equations that describe the processed afterglow spectrum, and in $\S 3$ we present numerical results for the time evolution of the associated absorption lines. Our discussion ignores recombination, since the recombination time $\left(\sim 10^{5} \mathrm{yr}_{1}^{-1}\right)$ is typically much longer than the lifetime of the afterglow. Our main conclusions are summarized in $\S 4$.

\section{IONIZATION DYNAMICS}

We consider a GRB source that turns on at time $t=0$ and illuminates a stationary ambient medium of uniform density $n$, with a time-dependent luminosity per unit frequency, $L_{v}(t)$. Since most of the interstellar absorption occurs at large radii, we ignore the initial subparsec fireball phase, during which the radiation is still coupled to the hydrodynamics. In the late phase of interest, the pressure

\footnotetext{
${ }^{1}$ Note that the observed time, which is related to the width of the outgoing radiation shell, it typically much shorter than the light crossing time of the absorber.
}

wave has already slowed down to subrelativistic speeds, so that it lags behind the radiation field and has no effect on the absorption spectrum of the outgoing radiation shell. The ionization state of the gas is then affected only by the luminosity $L_{v}(t)$ at frequencies higher than the ionization threshold $v_{0}$.

The photoionization rate per atom of species $a$ (=hydrogen, helium, or metals) is given by

$$
-\frac{1}{n_{a}(r, t)} \frac{d n_{a}(r, t)}{d t}=\int_{v_{0, a}}^{\infty} d v \frac{F_{v}(r, t)}{h v} \sigma_{\mathrm{bf}, a}(v),
$$

where $F_{v}(r, t)=L_{v}(t) / 4 \pi r^{2}$ is the flux at a radius $r$ from the source, and $\sigma_{\mathrm{bf}}(v)$ is the photoionization (bound-free) cross section at a photon frequency $v$. All photons are assumed to propagate radially away from the central source. Equation (1) determines the evolution of the number density of atoms, $n_{a}(t)$, from that of the flux, $F_{v}(r, t)$. This flux is absorbed in the medium through both bound-free and bound-bound transitions. The radiative transfer equation in spherical symmetry reads

$$
\begin{aligned}
\frac{1}{r^{2}} \frac{d\left[r^{2} F_{v}(r, t)\right]}{d r}= & -F_{v}(r, t) \sum_{a} n_{a}(r, t) \\
& \times\left[\sigma_{\mathrm{bf}, a}(v)+\sigma_{\mathrm{bb}, a}(v)\right],
\end{aligned}
$$

where $\sigma_{\mathrm{bb}}$ is the cross section for bound-bound absorption.

The coupled equations (1) and (2) determine the evolution and spatial distribution of the flux and the ionization fraction for a given set of initial and boundary conditions. We assume for simplicity that the source is embedded in a spherical gas cloud of uniform density and radius $R$, namely,

$$
n_{a}(t=0, r)= \begin{cases}n_{a, 0} & \text { for } r \leq R \\ 0 & \text { otherwise }\end{cases}
$$

and start the calculation at a radius $r_{0} \sim 10^{17} \mathrm{~cm}$, with the boundary condition

$$
F_{v}\left(r_{0}, t\right)=\frac{L_{v}(t)}{4 \pi r_{0}^{2}} .
$$

Our results are insensitive to the precise choice of $r_{0}$, as long as this radius is much smaller than the scale over which the ambient interstellar medium is distributed.

Following Waxman (1997a, 1997b), we model the time and frequency dependence of the afterglow luminosity at emission as

$$
L_{v}(t)=L_{v_{\mathrm{m}}}\left(\frac{v}{v_{\mathrm{m}}(t)}\right)^{-\alpha}
$$

where

$$
v_{m}(t)=1.7 \times 10^{16}\left(\frac{\xi_{e}}{0.2}\right)^{2}\left(\frac{\xi_{B}}{0.1}\right)^{1 / 2} E_{52}^{1 / 2} t_{\mathrm{hr}}^{-3 / 2} \mathrm{~Hz} .
$$

Here $\xi_{B}$ and $\xi_{e}$ are the fractions of the equipartition energy in magnetic field and accelerated electrons, $E=10^{52} E_{52}$ ergs is the fireball energy, $t_{\mathrm{hr}} \equiv(t / \mathrm{hr})$, and

$$
L_{v_{m}}=8.65 \times 10^{29} \sqrt{n_{1}}\left(\frac{\xi_{B}}{0.1}\right) E_{52} \operatorname{ergs~s}^{-1} \mathrm{~Hz}^{-1},
$$

where $n_{1}$ is the ambient proton density in units of $1 \mathrm{~cm}^{-3}$.

In our numerical examples, we use the typical parameter values that are required to fit the existing afterglow data, 
namely, $\xi_{e} E_{52}^{1 / 4}=0.2, \xi_{B}=0.1,\left(n_{1}\right)^{1 / 2} E_{52}=1, \alpha=0.5$ for $v>v_{m}$, and $\alpha=-1 / 3$ for $v<v_{m}$.

Finally, the atomic cross sections in equation (2) are calculated as follows.

1. Bound-Bound absorption. The cross section for transitions between two bound states $i$ and $k$ of an atom has a Lorentzian shape (Rybicky \& Lightman 1979, p. 289),

$$
\sigma_{\mathrm{bb}}^{i, k}=f_{i, k} \frac{3 \lambda_{k, i}^{2}}{8 \pi} \frac{\Lambda_{k, i}^{2}\left(v / v_{k, i}\right)^{2}}{4 \pi^{2}\left(v-v_{k, i}\right)^{2}+\Lambda_{k, i}^{2}\left(v / v_{k, i}\right)^{6} / 4},
$$

where $\lambda_{k, i}=c / v_{k, i}$ is the transition wavelength and $\Lambda_{k, i}$ is the transition probability, which is related to the absorption oscillator strength $f_{k, i}$ through

$$
\Lambda_{k, i}=6.670 \times 10^{15} \mathrm{~s}^{-1} \frac{g_{k} f_{i, k}}{g_{k}\left(\lambda_{k, i} / \AA\right)^{2}},
$$

where $g_{i}$ and $g_{k}$ are the statistical weights of the levels $i$ and $k$, respectively. The values of these parameters for permitted resonance lines of the most important astrophysical elements are summarized by Verner, Verner, \& Ferland (1996). For simplicity, we assume a cold, quiescent absorber. Thermal or turbulent broadening would change the line profile but not affect its time dependence, which is dictated by bound-free transitions (cf. eq. [1]).

2. Bound-Free absorption. An analytical expression for the photoionization cross section exists for single-electron atoms (Hall 1936), and for hydrogen reads

$$
\sigma_{b f, \mathrm{H}}(v)=A_{0}\left(\frac{v_{0}}{v}\right)^{4} \frac{\exp \left[4-\left(4 \tan ^{-1} \epsilon\right) / \epsilon\right]}{1-\exp (-2 \pi / \epsilon)},
$$

$$
\text { for } v \geq v_{0},
$$

where $A_{0}=6.30 \times 10^{-18} \mathrm{~cm}^{2}$ and $\epsilon=\left(v / v_{0}-1\right)^{1 / 2}$, where $v_{0}=3.29 \times 10^{15} \mathrm{~Hz}$ is the photoionization frequency threshold for an electron in the ground state. For simplicity, we assume that all neutral hydrogen atoms are in the ground state; the excitation rate to higher levels does not exceed the photoionization rate for the characteristic power-law spectrum of GRB afterglows.

For nonhydrogenic atoms, Verner et al. (1993) obtained a useful analytical fit to the photoionization cross section $\sigma_{n, l}(v)$ for an electron in a shell with quantum numbers $n, l$. The cross section can be written as $\sigma_{n, l}(v)=\sigma_{\star} F(y)$ for frequencies $v \geq v_{n, l}$, where $y \equiv v / v_{\star}$. Here $v_{n, l}$ is the threshold frequency for photoionization of an electron in the shell $n, l$; $\sigma_{\star}=\sigma_{\star}(n, l, Z, N)$ and $v_{\star}=v_{\star}(n, l, Z, N)$ are fitting parameters that depend on the atomic number $Z$ and number of electrons $N$, and $F(y)=\left[(y-1)^{2}+y_{w}^{2}\right] y^{-Q}[1$ $\left.+\left(y / y_{a}\right)^{1 / 2}\right]^{-P}$, where $Q=5.5+l-0.5 P$, and $y_{w}, y_{a}$, and $P$ are three additional fit parameters that depend on $n, l, Z$, and $N$. The total cross section to photoionization is obtained by summing over all shells,

$$
\sigma_{\mathrm{bf}}(v)=\sum_{n, l} \sigma_{n, l}(v)
$$

We ignore secondary ionization by fast electrons created by $\mathrm{X}$-ray or gamma-ray photoionization.

\section{NUMERICAL RESULTS: TIME DEPENDENCE OF ABSORPTION LINES}

Since hydrogen is by far the most abundant element in galaxies, we examine first its prominent Ly $\alpha$ absorption signature. Figure 2 shows the expected evolution of the after- glow spectrum in a narrow frequency band around the Ly $\alpha$ resonance for an $\mathrm{H}$ I column density of $3 \times 10^{20} \mathrm{~cm}^{-2}$ and two different cases of spherical absorbing clouds around the source: (1) a compact $(R=1 \mathrm{pc})$ and dense $\left(n_{\mathrm{H}, 0}=100\right.$ $\mathrm{cm}^{-3}$ ) cloud (Fig. 2, left panels), and (2) a more extended $(R=100 \mathrm{pc})$ and rarefied $\left(n_{\mathrm{H}, 0}=1 \mathrm{~cm}^{-3}\right)$ cloud (Fig. 2, right panels). The first case illustrates the conditions in a molecular cloud, while the second corresponds to a galactic disk of scale height $\sim 100$ pc. Although the two spectra initially possess the same absorption profile, because of their identical column density, their time evolution is very different. The smaller absorber is ionized faster, because its atoms are exposed to a higher ionizing flux and because it requires fewer photons to get ionized as a consequence of its smaller mass.

The photoionization efficiency can be quantified through the change in the equivalent width (EW) of the absorption line, defined as

$$
\mathrm{EW}=\int d \lambda \frac{\left(F_{v, 0}-F_{v}\right)}{F_{v, 0}},
$$

where $F_{v}(\lambda)$ is the observed spectral flux (at $r \gg R$ ) as a function of wavelength across the line, and $F_{v, 0}(\lambda)$ is the continuum flux that the afterglow would have had if there were no absorption. The values of the EW for the Ly $\alpha$ line are listed in the different panels of Figure 2. For a compact absorbing region such as a molecular cloud, the EW could decrease by orders of magnitude within several days after the GRB detection.

Although ground-based observations are not capable of detecting Ly $\alpha$ absorption for absorber redshifts $z \lesssim 2$, they can still probe metal absorption lines, as demonstrated in the case of GRB 970508 (Metzger et al. 1997; Djorgovski et al. 1997). Because metal lines are often unsaturated, it is easier to detect the time evolution in their case than in the damped Ly $\alpha$ trough. Figure 3 illustrates the expected evolution of the $\mathrm{Mg}$ II absorption line at $\lambda_{0}=2798 \AA$, which was detected in the spectrum of GRB $970508 .^{2}$ Our calculations assume a solar abundance of $\mathrm{Mg}$ II (Anders \& Grevesse 1989). Since there are far fewer $\mathrm{Mg}$ II atoms than $\mathrm{H}$ I atoms, it takes fewer photons and less time from the onset of the afterglow to ionize them. Therefore, the evolution of the metal-line EW in Figure 3 is more rapid than that of the Ly $\alpha$ EW in Figure 2.

In Figure 4 we show a broader range of the spectrum, including the UV and X-ray regimes, where flux is absorbed by photoionization of hydrogen. For a $\sim 1$ pc absorber, the absorption profile narrows considerably after a couple of weeks. Consequently, the observed flux from a source at a redshift $z_{s}=1$ in the emission band of $13.6-150 \mathrm{eV}$ increases from $10^{-11}$ ergs $\mathrm{cm}^{-2} \mathrm{~s}^{-1}$ after $6 \mathrm{hr}$ to $4.2 \times 10^{-11}$ ergs $\mathrm{cm}^{-2} \mathrm{~s}^{-1}$ after 2 days, despite the overall fading of the afterglow emission.

All of the above time dependences occur only for absorbers that are associated with the GRB source. More distant absorbers along the line of sight are exposed to an afterglow flux that is much too weak to affect their ioniza-

\footnotetext{
${ }^{2}$ Note that if $\mathrm{Mg}$ II absorption occurs primarily in galactic halos, as implied by quasar absorption studies (Steidel \& Sargent 1992), then it is less likely to be associated with star-forming regions such as galactic disks. In that case, it might be more appropriate to monitor lines that originate in cooler environments, such as $\mathrm{Mg}$ I.
} 


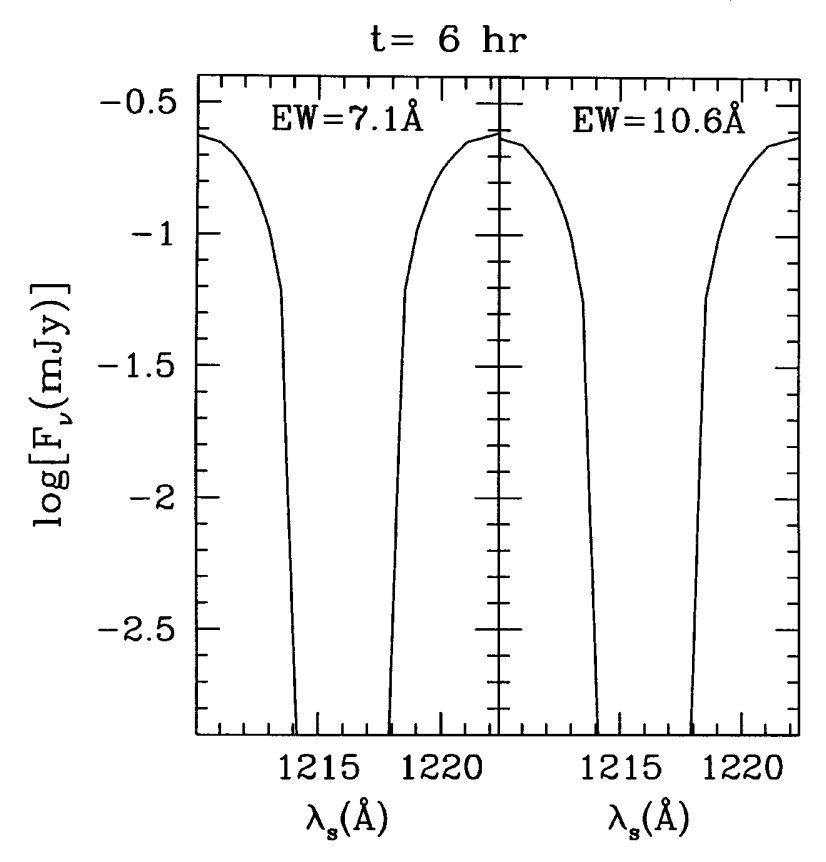

Ly $\alpha$
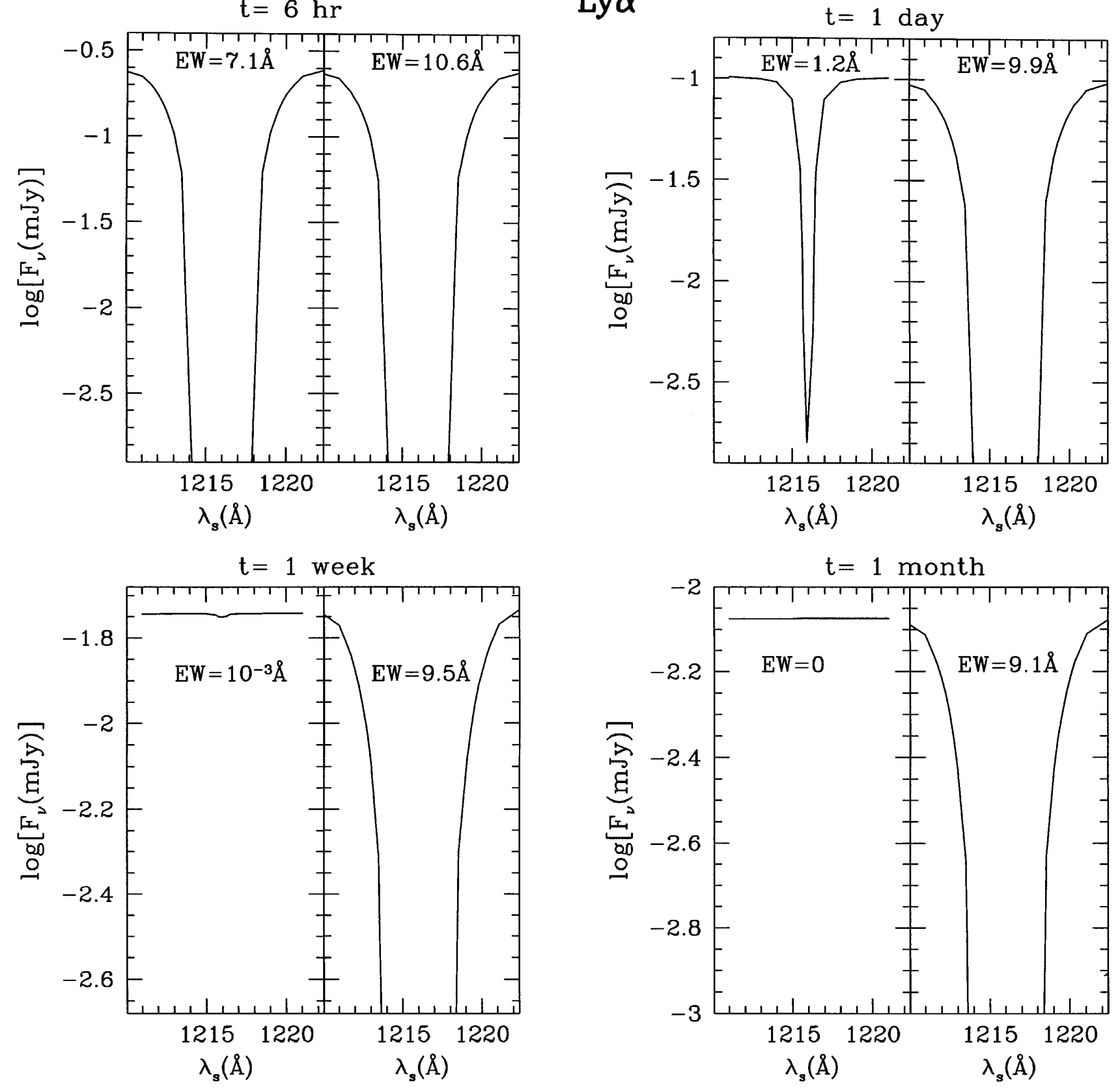

Fig. 2.-Evolution of the observed Ly $\alpha$ absorption profile for a spherical gas cloud around a GRB source, with a radius $R=1 \mathrm{pc}$ and an initial $\mathrm{H}$ I density of $n_{\mathrm{H}, 0}=100 \mathrm{~cm}^{-3}$ (left panels), or with $R=100 \mathrm{pc}$ and $n_{\mathrm{H}, 0}=1 \mathrm{~cm}^{-3}$ (right panels). The equivalent width (EW) of the line is listed in each case. The vertical axis shows the observed flux for a source redshift $z_{s}=1$ (for an $h=0.5, \Omega=1$ cosmology), and the horizontal axis shows the emission wavelength at the source, $\lambda_{s}=\lambda /\left(1+z_{s}\right)$.

tion state during the brief period of time over which the radiation lasts.

If the GRB environment is metal rich, then it is likely to also contain dust. Broadband extinction by dust would suppress the observed optical-UV flux from the afterglow by a wavelength-dependent factor. The deviation from the fireball predictions would weaken at late times as a result of the heating of dust above its sublimation temperature by the afterglow radiation (Waxman \& Phinney 1998); this process might explain the transient suppression of optical relative to X-ray emission and the relative reddening during the first 2 days of GRB 970508. The absence of an optical afterglow for GRB 970828 might also have been caused by dust extinction (Groot et al. 1998). This interpretation is supported by the low energy turnover in the ASCA X-ray spectrum of GRB 970828, which implies a high hydrogen column density $\left(\sim 10^{21}-10^{22} \mathrm{~cm}^{-2}\right)$ along the line of sight to the source (Murakami et al. 1997). Figure 4 indicates that if this absorption originated from a compact molecular cloud around the GRB source, then the X-ray absorption feature must have evolved on a timescale of days. For a given inferred column density, the level of evolution becomes weaker with increasing absorber mass.

\section{CONCLUSIONS}

Afterglow models postulate the existence of gas-rich environments around some GRB sources. We have shown that this conjecture can be tested empirically by monitoring 


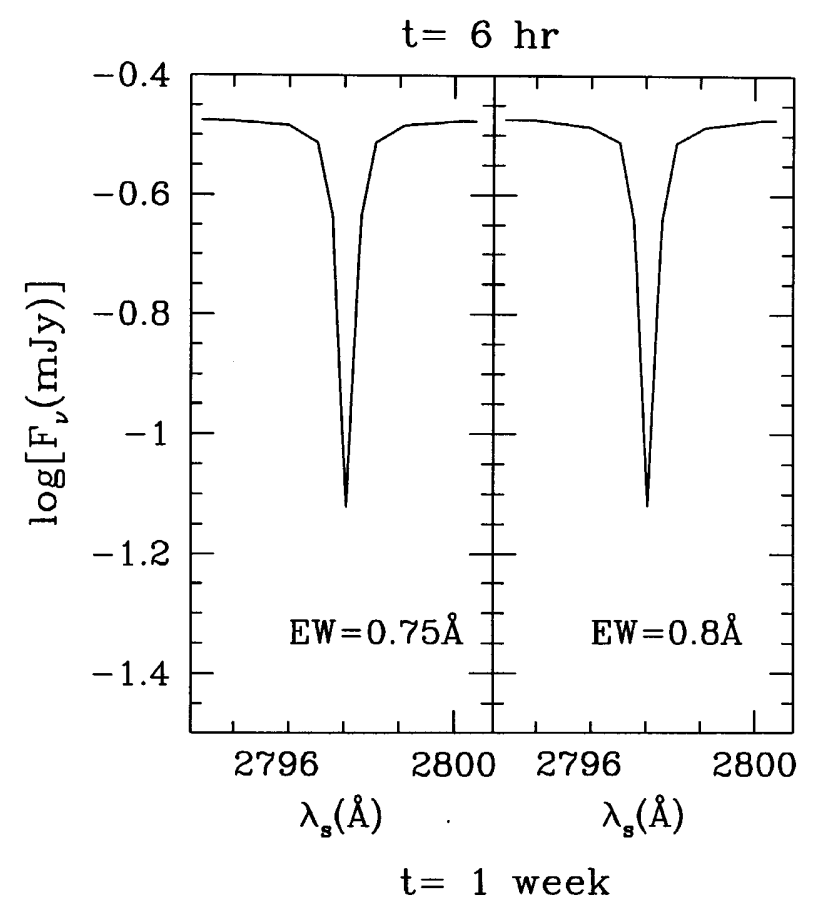

MgII
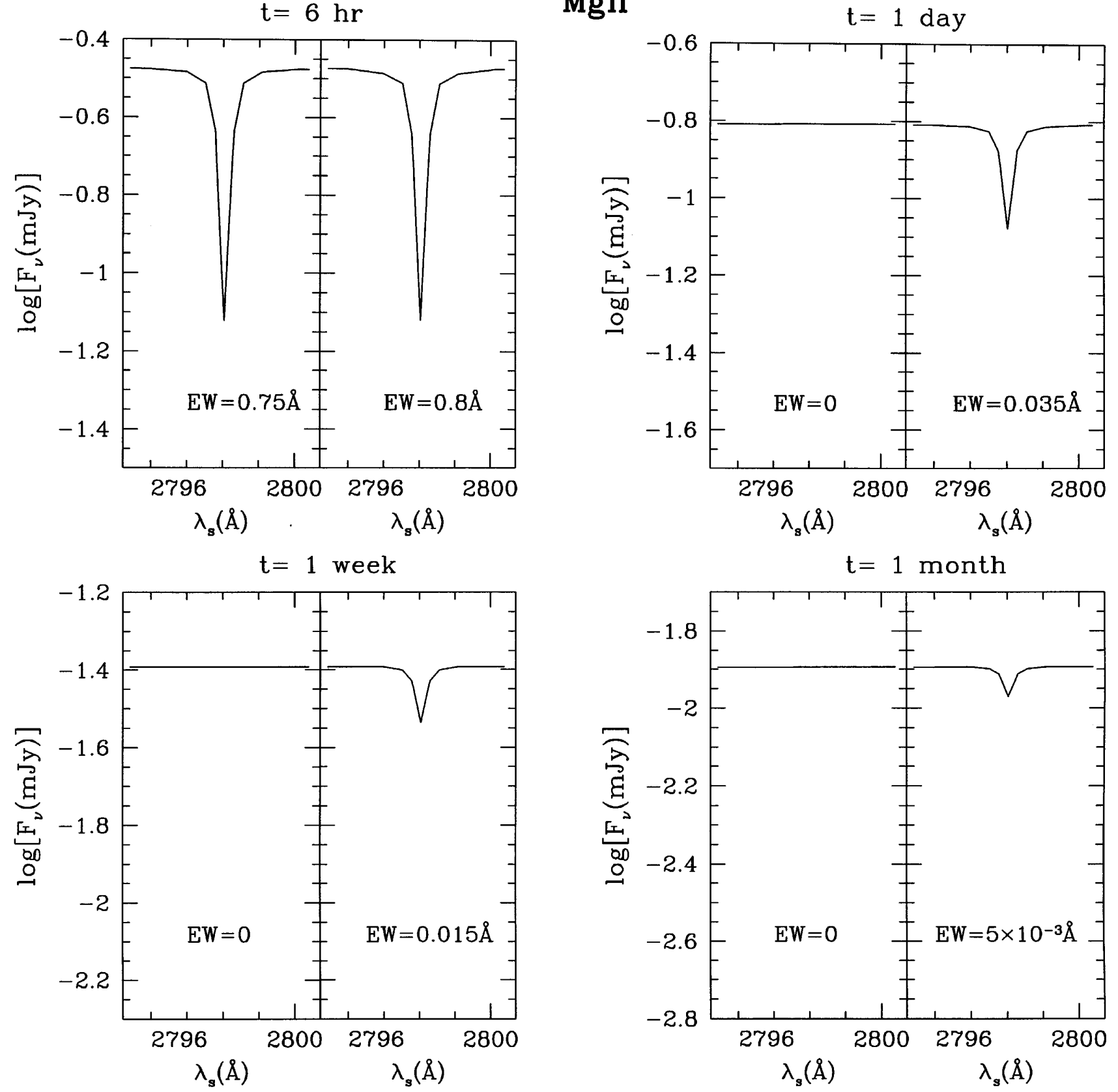

FIG. 3.-Evolution of the observed $\mathrm{Mg}$ II absorption line for a spherical gas cloud of solar metallicity, with $R=10 \mathrm{pc}$ and an initial hydrogen density of $n_{\mathrm{H}, 0}=10 \mathrm{~cm}^{-3}$, or equivalently a $\mathrm{Mg}$ II density $n_{\mathrm{Mg}, 0}=3.8 \times 10^{-4} \mathrm{~cm}^{-3}$ (left panels), as compared to a cloud with $R=100 \mathrm{pc}, n_{\mathrm{H}, 0}=1 \mathrm{~cm}{ }^{-3}$, and $n_{\mathrm{Mg}, 0}=3.8 \times 10^{-5} \mathrm{~cm}^{-3}$ (right panels). Axis notations are the same as in Fig. 2.

the afterglow absorption spectra. If the surrounding gas is not fully ionized initially, then the bright afterglow radiation will modify its ionization state. The medium into which the afterglow radiation propagates at late times would be more ionized than the medium into which the early radiation propagated. Hence, the equivalent width (EW) of the associated absorption lines would decline with time during the afterglow (see Fig. 2). The rate of EW variation can be used to constrain the size of the absorbing region; the more compact the absorbing region around the GRB source, the higher the photon flux to which it is exposed, and the faster it gets ionized. When combined with the value of the column density inferred from the EW measurement itself, the size estimate can be used to find the density of the ambient medium. ${ }^{3}$ One could then test statistically the predicted correlation between the afterglow flux and this density (cf. eq. [7]). In principle, it might even be possible to infer the profile of a nonuniform density distribution along the line of sight based on frequent monitoring of the EW evolution. Identification of time dependence in the afterglow spectrum would isolate the variable (and hence associated) absorption lines from other lines that are caused by chance intersections of unrelated absorbers along

\footnotetext{
${ }^{3}$ Note that if the absorber size or density can be measured independently by another method, then it might be possible to infer the luminosity distance to the source and by that constrain the values of cosmological parameters.
} 

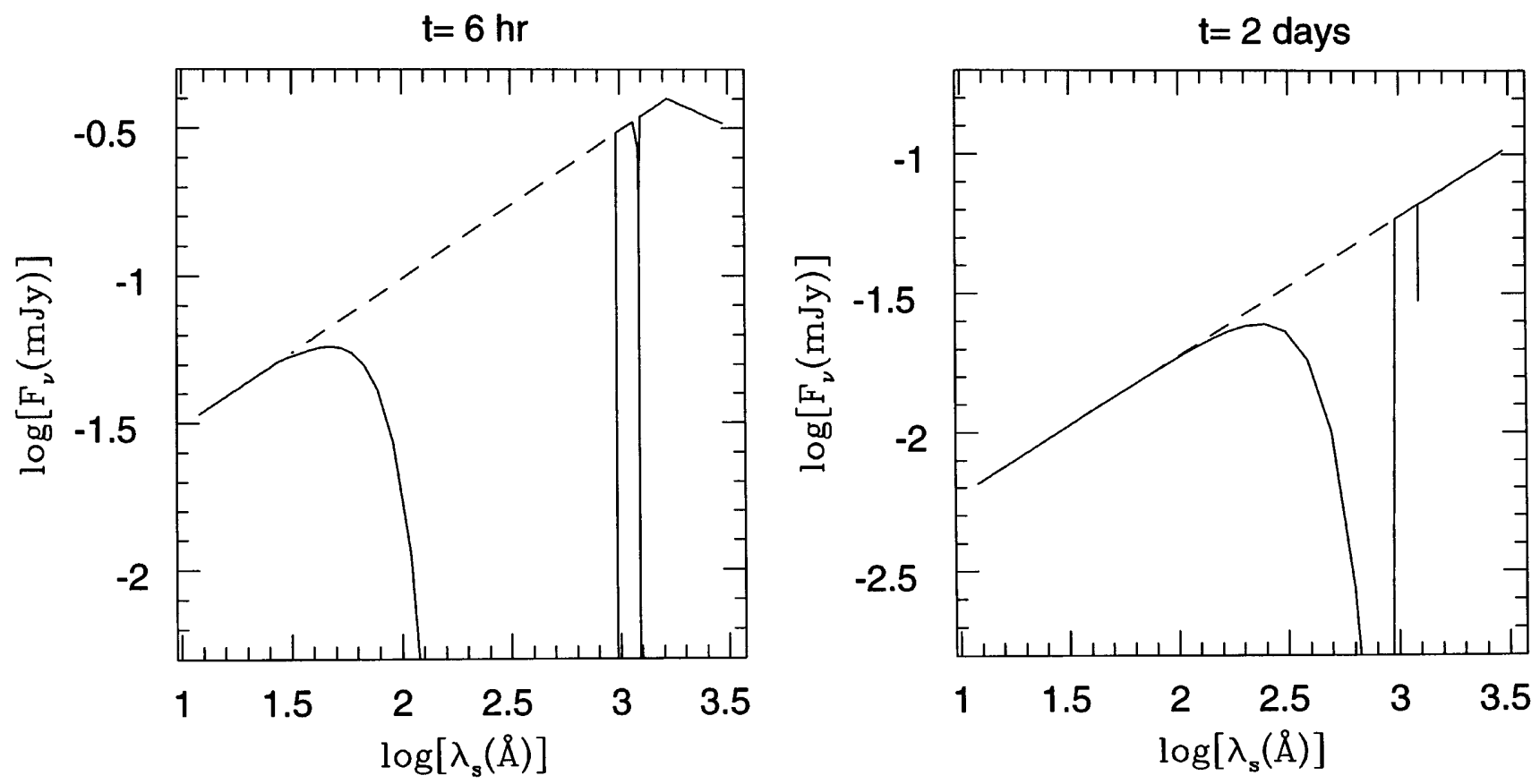

FIG. 4.-Evolution of the soft X-ray and UV absorption for a spherical gas cloud with $R=1 \mathrm{pc}$ and an initial $\mathrm{H} \mathrm{I}$ density of $n_{\mathrm{H}, 0}=100 \mathrm{~cm}^{-3}$. The dashed line shows the afterglow flux that would have been observed if there were no absorption. Axis notations are the same as in Fig. 2.

the line of sight, and hence provide a precise determination of the GRB redshift.

Our results imply that if the observed metal absorption lines of GRB 970508 (Metzger et al. 1997; Djorgovsky et al. 1997 ) occurred in a region of radius $\lesssim 100$ pc around the GRB source itself, then the equivalent width of the lines would have declined noticeably over the first month of the afterglow (Fig. 3). Similarly, the soft X-ray absorption that was possibly detected by $A S C A$ in GRB 970828 (Murakami et al. 1997) should have decayed under the same conditions (Fig. 4). Detection of these effects in future GRBs would help determine the properties of their gaseous environments and their redshifts.

Since the peak optical fluxes of afterglows such as GRB 970508 are $\gtrsim 10^{2}$ times brighter than supernovae, they can be used to probe galactic environments at exceedingly high redshifts. If the GRB source population traces the star formation history of the universe, then the dimmest GRB events known might originate from a redshift as high as $z \sim 6$ (Wijers et al. 1997a). In hierarchical cold dark matter models of structure formation (e.g., Navarro, Frenk, \& White 1997), high-redshift hosts are expected to be denser $\left[n \propto\left(1+z_{f}\right)^{3}\right]$ and more compact $\left[R \propto M^{1 / 3}\left(1+z_{f}\right)^{-1}\right.$, where $z_{f}$ is their (higher) formation redshift, and $M$ is their (smaller) mass] than low-redshift galaxies, hence making the effects discussed in this paper even more pronounced.

We thank Ue-Li Pen, John Raymond, Dimitar Sasselov, and Eli Waxman for useful discussions, and Sarah Jaffe and Max Poletto for help in the preparation of the figures. We are also grateful to the referee, Andy Fruchter, for insightful comments. This work was supported in part by a graduate student fellowship from the University of Salerno, Italy (for R. P.), and by NASA ATP grant NAG5-3085 and the Harvard Milton fund (for A. L.).
Anders, E., \& Grevesse, N. 1989, Geochim. Cosmochim. Acta, 53, 197 Bond, H. E. 1997, IAU Circ. No. 6654

Blandford, R. D., \& Mckee, C. F. 1976, Phys. Fluids, 19, 1130

Broeils, A. H., \& van Woerden, H. 1994, A\&AS, 107, 129

Costa, E., et al. 1997, Nature, 387, 783

Djorgovski, S. G., et al. 1997, Nature, 387, 876

Frail, D. A., et al. 1997, Nature, in press

Fruchter, A., et al. 1997, IAU Circ. No. 6747

Goodman, J. 1997, New Astron., 2, 449

Groot, P. J., et al. 1998, ApJ, 493, L27

Hall, H. 1936, Rev. Mod. Phys., 8, 358

Meszaros, P.\& Rees, M. J. 1997, 476, 232

Metzger, M. R., et al. 1997, Nature, 387, 878

Murakami, T., et al. 1997, in Proc. 4th Huntsville Symp., ed. C. A. Meegan, R. Preece, \& T. M. Koshut (in press)

Navarro, J., Frenk, C. S., \& White, S. D. M. 1997, ApJ, 490, 493

Paczyński, B., \& Rhoads, J. E. 1993, ApJ, 418, L5

Pian, E., et al. 1997, in Proc. 4th Gamma-Ray Burst Symp., ed. C. A. Meegan, R. Preece, \& T. Koshut (in press)

Rybicky, G. B., \& Lightman, A. P. 1979, Radiative Processes in Astrophysics (New York: Wiley)

\section{REFERENCES}

Sahu, K., et al. 1997, ApJ, 489, L127

Sari, R. 1997, ApJ, 489, L37

Sari, R., Piran, T., \& Narayan, R. 1997, preprint astro-ph/9712005

Steidel, C. C., \& Sargent, W. L. W. 1992, ApJS, 80, 1

Taylor, G. B., Frail, D. A., Beasley, A. J., \& Kulkarni, S. R. 1997, Nature, 389,263

van Paradijs, J., et al. 1997, Nature, 386, 686

Verner, D. A., Verner, E. M., \& Ferland, G. J. 1996, At. Data Nucl. Data Tables, 64, 1

Verner, D. A., Yakolev, D. G., Band, L. M., \& Trzhaskovskaya, M. B. 1993,

At. Data Nucl. Data Tables, 55, 234

Vietri, M. 1997a, ApJ, 478, L9

. 1997b, ApJ, 488, L105

Waxman, E. 1997a, ApJ, 485, L5

.1997b, ApJ, 489, L33

Waxman, E., Kulkarni, S. R., \& Frail, D. 1998, ApJ, 497, 288

Waxman, E., \& Phinney, E. S. 1998, in preparation

Wijers, R. A. M. J., Bloom, J. S., Bagla, J. S., \& Natarajan, P. 1997a, MNRAS, submitted (preprint astro-ph/9708183)

Wijers, R. A. M. J., Rees, M. J., \& Meszaros, P. 1997b, MNRAS, 288, L51 